\title{
A Comparison of \\ Projection Operator Formalisms for the Study of Self-Diffusion
}

\author{
R. D. Mosteller ${ }^{1,2}$ and J. J. Duderstadt ${ }^{1}$
}

Received April 18, 1973

The utility of projection operator formalisms for describing the dynamics of many-body systems is studied, and the compatibility of these formalisms with certain approximation schemes is evaluated in the light of known behavior of such systems. For simplicity the investigation is limited to the study of Brownian motion. Specifically, a memory kernel formalism and a kinetic equation formalism are compared for the calculation of the time evolution of the momentum autocorrelation function. Both perturbation expansions and averaged propagator approximations are investigated. The results from these studies suggest that the long-time behavior of the momentum autocorrelation function is sensitive to the long-range nature of the interparticle potential.

KEY WORDS: Brownian motion; kinetic equation; Markovian approximations; memory kernel equation; momentum autocorrelation function; projection operator formalisms; self-diffusion.

\section{INTRODUCTION}

Projection operator techniques have proven extremely useful in formal studies of the dynamics of many-body systems. To date, however, their

This work was supported in part by the National Science Foundation under Grant GK19360X.

${ }^{1}$ Department of Nuclear Engineering, The University of Michigan, Aun Arbor, Michigan.

' Present address: Department of Nuclear Science and Engineering, The Catholic University of America, Washington, D.C. 
main applications in specific calculations have been limited to attempts to develop phenomenological models of nonequilibrium behavior. More fundamental investigations, of course, would rely upon the introduction of mathematical approximations-the familiar perturbation approximations in coupling and density, for example - directly into the projection operator formalism. The utility of projection operator methods within this context was dealt a blow by the observation of Resibois et al..$^{(1)}$ that more rigorous approaches such as perturbation theory frequently are not compatible with these methods. This and other recent investigations have cast some doubt on the efficacy of projection operator methods for investigations other than those which are purely formal or which involve specific phenomenological models.

The objectives of this paper are to study and compare the various ways in which approximations can be introduced into projection operator formalisms and to evaluate these approximations in the light of known behavior of many-body systems. For simplicity these techniques are applied to the study of Brownian motion, which constitutes the most elementary problem in nonequilibrium statistical mechanics. In particular, two alternative projection operator formalisms are studied and compared for the calculation of the momentum autocorrelation function

$$
\Phi(t) \equiv\left(1 / 3 m k_{B} T\right)\left\langle\mathbf{p}_{1} \cdot \mathbf{p}_{1}(t)\right\rangle \equiv(\beta / 3 m)\left\langle\mathbf{p}_{1} \cdot \mathbf{p}_{1}(t)\right\rangle
$$

which characterizes the motion of a test particle with mass $m$ and initial momentum $\mathbf{p}_{1}$ in a three-dimensional $N$-body system. The first formalismthe memory kernel formalism (MKF)--is based upon a hydrodynamics-like equation for $\Phi(t)$ itself, while the other-the kinetic equation formalism $(\mathrm{KEF})$ - is based upon a kinetic equation for its phase-space generalization, $\boldsymbol{\Theta}(\mathbf{p}, t)$.

The relation between the two levels of description inherent in these formalisms is explored in some detail. It is found that certain classes of approximations for the modified propagator which appears in these equations (in particular, low-order perturbation approximations) yield incorrect results in one formalism but valid results in the other. Further, it is shown that the difference in the two formalisms is due to the presence of an additional projection operator in the MKF. This operator removes the $\mathbf{p}$-space dependence from the memory kernel description and thereby causes it to yield invalid results in the long-time limit of certain perturbation approximations. The relationship between the two formalisms is demonstrated even more clearly by means of a variational principle for the KEF. Within the context of a first-order perturbation approximation the kinetic description reduces to the memory kernel description when $\Theta(p, t)$ is approximated by its initial value $\mathbf{p} M(\mathbf{p})$, where $M(\mathbf{p})$ is the Maxwellian distribution in momentum. 
The usual perturbation expansions in coupling and density are discussed within the context of test particle motions, and an additional perturbation scheme is studied which reduces the kinetic equation for $\boldsymbol{\theta}(\mathbf{p}, t)$ to the familiar linearized Balescu-Lennard equation at long times. In addition, alternative approximation schemes are investigated which replace the modified propagator by "averaged" propagators, specifically the LorentzBoltzmann operator and the linearized Boltzmann operator. The success of these approximations in describing the known short- and long-time behavior of $\Phi(t)$ is investigated, with particular emphasis upon the nonhydrodynamic long-time behavior recently revealed by computer simulations of molecular dynamics.

\section{A FORMAL COMPARISON OF THE MKF AND THE KEF}

The time evolution of the momentum autocorrelation function $\Phi(t)$ can be described exactly by a memory kernel equation, ${ }^{(2)}$

$$
\dot{\Phi}(t)+\int_{0}^{t} d \tau K(t-\tau) \Phi(\tau)=0
$$

The memory kernel $K(t)$ is given formally as

$$
K(t) \equiv(\beta / 3 m)\left\langle\mathbf{F}_{\mathbf{1}} \cdot e^{i t\left(1-\mathscr{O}_{M}\right) L} \mathbf{F}_{\mathbf{1}}\right\rangle
$$

where $F_{1}$ is the initial force on the test particle and $L$ is the well-known Liouville operator, $i L_{0} \equiv\{H, \circ\}_{P_{B}}$. The projection operator $\mathscr{P}_{M}$ was first introduced by Zwanzig ${ }^{(2-4)}$ and Mori $^{(5)}$ and is defined by its action upon a general phase-space variable $\mathbf{G}$,

$$
\mathscr{P}_{M} \mathbf{G} \equiv(\beta / 3 m) \mathbf{p}_{\mathbf{1}}\left\langle\mathbf{p}_{\mathbf{1}} \cdot \mathbf{G}\right\rangle
$$

The memory kernel equation (2) corresponds to a generalized hydrodynamic (i.e., configuration-space) description of $\Phi(t)$.

An alternative but still exact description of the momentum autocorreIation function can be given in terms of a generalized kinetic equation

$$
\dot{\Theta}(\mathbf{p}, t)+\int_{0}^{t} d \tau \int d^{3} p^{\prime \prime} \varphi\left(\mathbf{p}, \mathbf{p}^{\prime}, t-\tau\right) \boldsymbol{\Theta}\left(\mathbf{p}^{\prime}, \tau\right)=0
$$

where $\boldsymbol{\Theta}(\mathbf{p}, t)$ is a phase-space generalization of $\Phi(t)$,

$$
\Phi(t)=\int d^{3} p \mathbf{p} \cdot \mathbf{\Theta}(\mathbf{p}, t)
$$

The collision kernel $\varphi\left(\mathbf{p}, \mathbf{p}^{\prime}, t\right)$ is given formally as

$$
\varphi\left(\mathbf{p}, \mathbf{p}^{\prime}, t\right) \equiv \frac{1}{M\left(\mathbf{p}^{\prime}\right)}\left\langle\mathbf{F}_{1} \cdot\left[\frac{\hat{\partial}}{\partial \mathbf{p}_{1}} \delta\left(\mathbf{p}^{\prime}-\mathbf{p}_{1}\right)\right] e^{i t\left(1-\mathscr{P}_{K^{\prime}} L \mathbf{F}_{1}\right.} \cdot \frac{\partial}{\partial \mathbf{p}_{1}} \delta\left(\mathbf{p}-\mathbf{p}_{1}\right)\right\rangle
$$


where the projection operator $\mathscr{P}_{K}$ is defined by

$$
\begin{aligned}
\mathscr{P}_{K} \mathbf{G} & \equiv \int d^{3} p^{\prime}\left[1 / M\left(\mathbf{p}^{\prime}\right)\right]\left\langle\mathbf{G} \delta\left(\mathbf{p}^{\prime}-\mathbf{p}_{1}\right)\right\rangle \delta\left(\mathbf{p}^{\prime}-\mathbf{p}_{1}\right) \\
& =\int d \Gamma_{B} \int d^{3} x_{1}\left[f_{0}\left(\Gamma_{N}\right) / M\left(\mathbf{p}_{1}\right)\right] \mathbf{G}\left(\Gamma_{N}\right)
\end{aligned}
$$

$f_{0}$ denotes the equilibrium phase-space distribution function for the system, while $\Gamma_{N}$ and $\Gamma_{B}$ are the ensemble variables for the system and the bath, respectively; i.e., $\Gamma_{N} \equiv\left\{\mathbf{x}_{1}, \mathbf{x}_{2}, \ldots, \mathbf{p}_{N}, \mathbf{p}_{1}, \mathbf{p}_{2}, \ldots, \mathbf{p}_{N}\right\}$ and $\Gamma_{B} \equiv\left\{\mathbf{x}_{2}, \ldots\right.$, $\left.\mathbf{x}_{N}, \mathbf{p}_{2}, \ldots, \mathbf{p}_{N}\right\}$.

The relationship between $\mathscr{P}_{M}$ and $\mathscr{P}_{K}$ can be demonstrated most easily by first defining a new projection operator $\mathscr{P}_{1}$,

$$
\mathscr{P}_{\mathbf{1}} \mathbf{G} \equiv(\beta / 3 m) \mathbf{p}_{1} \int d^{3} \hat{p}_{1} M\left(\hat{\mathbf{p}}_{1}\right) \hat{\mathbf{p}}_{1} \cdot \mathbf{G}\left(\hat{\Gamma}_{N}\right)
$$

It is then apparent that

$$
\begin{aligned}
\mathscr{P}_{1} \mathscr{P}_{K} \mathbf{G} & =\frac{\beta}{3 m} \mathbf{p}_{1} \int d^{3} \hat{p}_{1} M\left(\hat{\mathbf{p}}_{\mathbf{1}}\right) \int d^{3} x_{\mathbf{1}} \int d \Gamma_{B}\left[\frac{f_{\mathbf{0}}\left(\Gamma_{N}\right)}{M\left(\hat{\mathbf{p}}_{1}\right)}\right] \hat{\mathbf{p}}_{1} \cdot \mathbf{G}\left(\hat{\Gamma}_{N}\right) \\
& =\mathscr{P}_{M} \mathbf{G}=\mathscr{P}_{K} \mathscr{P}_{1} \mathbf{G}
\end{aligned}
$$

Such a result is not surprising, since the memory kernel equation (2) involves only configuration-space variables. The effect of the projection operator $\mathscr{P}_{1}$ is merely to remove an additional momentum dependence from the dynamic variable upon which it acts.

A further comparison between the two formalisms may be made directly in terms of two generalized collision operators. Prior to such a comparison it is convenient to introduce a Laplace transformation in time,

$$
\widetilde{\Phi}(s)=\int_{0}^{\infty} d t e^{-s t} \Phi(t)
$$

Next define a "collision operator" $\mathscr{J}_{K E}$ such that

$$
\mathscr{J}_{K E} \mathbf{G}(\mathbf{p}) \equiv \int d^{3} p^{\prime} \tilde{\varphi}\left(\mathbf{p}, \mathbf{p}^{\prime}, s\right) \mathbf{G}\left(\mathbf{p}^{\prime}\right)
$$

and finally define another operator $\mathscr{J}_{M K}$,

$$
\mathscr{J}_{M K} \mathbf{G} \equiv \mathscr{P}_{K} L\left[s-i\left(1-\mathscr{P}_{K} \mathscr{P}_{1}\right) L\right]^{-1} L \mathbf{G}
$$

such that

$$
\tilde{K}(s)=(\beta / 3 m) \int d^{3} p_{1} M\left(\mathbf{p}_{1}\right) \mathbf{p}_{1} \cdot \mathscr{J}_{M K} \mathbf{p}_{1}
$$

The Laplace transform of Eq. (2) is just

$$
\widetilde{\Phi}(s)=1 /[s+\widetilde{K}(s)]
$$


which, in view of Eq. (14), may be rewritten as

$$
\widetilde{\Phi}(s)=\left\{(\beta / 3 m) \int d^{3} p_{1} M\left(\mathbf{p}_{1}\right) \mathbf{p}_{1} \cdot\left[s+\mathscr{J}_{M K}\right] \mathbf{p}_{1}\right\}^{-1}
$$

Similarly, the Laplace transform of Eq. (5) is

$$
s \tilde{\Theta}(\mathbf{p}, s)+\int d^{3} p^{\prime} \tilde{\varphi}\left(\mathbf{p}, \mathbf{p}^{\prime}, s\right) \tilde{\Theta}\left(\mathbf{p}^{\prime}, s\right)=\mathbf{p} M(\mathbf{p})
$$

This last equation, in conjunction with Eqs. (6) and (12), implies that

$$
\widetilde{\Phi}(s)=(\beta / 3 m) \int d^{3} p \mathbf{p} \cdot\left\{1 /\left(s+\mathscr{J}_{K E}\right)\right] \mathbf{p} M(\mathbf{p})
$$

It is straightforward, however, to show that

$$
\mathscr{J}_{K E} M(\mathbf{p})=\mathscr{J}_{M K} M\left(\mathbf{p}_{1}\right)=0
$$

Consequently, Eq. (18) may be rewritten as

$$
\widetilde{\Phi}(s)=(\beta / 3 m) \int d^{3} p M(\mathbf{p}) \mathbf{p} \cdot\left[1 /\left(s+\mathscr{J}_{K E}\right)\right] \mathbf{p}
$$

which makes the constrast with Eq. (16) all the more apparent. In the MKF the momentum autocorrelation function can be expressed as the reciprocal of an integral over an operator, while in the KEF it can be expressed as an integral over the inverse of a similar operator.

\section{A VARIATIONAL PRINCIPLE FOR THE KEF}

Further insight into the relationship between the two formalisms may be gained from a variational formulation of the KEF. Let a scalar product be defined between any two functions of momentum $\mathbf{h}_{1}$ and $\mathbf{h}_{2}$ by

$$
\left(\mathbf{h}_{1}, \mathbf{h}_{2}\right) \equiv \int d^{3} p M(\mathbf{p}) \mathbf{h}_{1}^{*}(\mathbf{p}) \cdot \mathbf{h}_{2}(\mathbf{p})
$$

Then, since the collision kernel $\varphi\left(\mathbf{p}, \mathbf{p}^{\prime}, t\right)$ satisfies the detailed balance relation

$$
M\left(\mathbf{p}^{\prime}\right) \varphi\left(\mathbf{p}, \mathbf{p}^{\prime}, t\right)=M\left(\mathbf{p}^{\prime}\right) \varphi\left(\mathbf{p}^{\prime}, \mathbf{p}, t\right)
$$

(see the appendix), the operators $\mathscr{J}_{M K}$ and $\mathscr{J}_{K E}$ are self-adjoint with respect to this scalar product for real $s$; e.g.,

$$
\left(\mathbf{h}_{1}, \mathscr{J}_{K E} \mathbf{h}_{2}\right)=\left(\mathscr{J}_{K E} \mathbf{h}_{1}, \mathbf{h}_{2}\right)
$$

Equations (20) and (21) imply that the momentum autocorrelation function may be expressed as

$$
\widetilde{\Phi}(s)=(\beta / 3 m)(\mathbf{p}, \tilde{\theta})
$$


where $\tilde{\theta}(\mathbf{p}, s)$ is the solution to the equation

$$
s \tilde{\theta}(\mathbf{p}, s)+\mathscr{J}_{K E} \tilde{\theta}=\mathbf{p}
$$

If $s$ is temporarily restricted to real values, Eq. (25) is a self-adjoint, inhomogeneous equation. Further, Eq. (24) does not require that $\tilde{\theta}(\mathbf{p}, s)$ be found explicitly-only its scalar product with $\mathbf{p}$ is needed. A variational principle based upon the so-called Schwinger functional (see, e.g., Ref. 6) is quite useful at this point. When applied to Eq. (25) this functional takes the form

$$
\mathscr{F}[\mathbf{v}]=|(\mathbf{p}, \mathbf{v})|^{2} /\left(\mathbf{v},\left[s+\mathscr{J}_{K E}\right] \mathbf{v}\right)
$$

For real $s, \mathscr{F}$ has an extremum at the point $\mathbf{v}=\tilde{\theta}$. Further,

$$
\mathscr{F}[\tilde{\boldsymbol{\theta}}]=(\mathbf{p}, \tilde{\theta})=(3 m / \beta) \tilde{\Phi}(s)
$$

Hence, this functional can be used to estimate $\widetilde{\Phi}(s)$ for real $s$ simply by choosing various trial functions for $\mathbf{v}$, and the resulting expressions then may be analytically continued into the complex $s$ plane. In addition, this scheme will yield $\tilde{\Phi}(s)$ to second order in the trial function error.

Consider in particular the simple trial function

$$
\mathbf{v} \rightarrow \mathbf{p}
$$

which corresponds to approximating $\boldsymbol{\Theta}(\mathbf{p}, s)$ by its initial value $\mathbf{p} M(\mathbf{p})$. It is evident that

$$
\mathscr{F}[\mathbf{p}]=\left\{\int d^{3} p M(\mathbf{p}) \mathbf{p} \cdot\left[s+\mathscr{J}_{K E}\right] \mathbf{p}\right\}^{-1}
$$

which in turn implies that

$$
\widetilde{\Phi}(s) \rightarrow\left\{(\beta / 3 m) \int d^{3} p M(\mathbf{p}) \mathbf{p} \cdot\left[s+\mathscr{J}_{K E}\right] \mathbf{p}\right\}^{-1}
$$

This result is very similar to the expression for $\widetilde{\Phi}(s)$ in the MKF, Eq. (16). The only difference in the two equations is that $\mathscr{I}_{K E}$ appears in Eq. (30) rather than $\mathscr{J}_{M K}$.

As the next section indicates, however, many approximations for the modified propagators $\left[s-i\left(1-\mathscr{P}_{M}\right) L\right]^{-1}$ and $\left[s-i\left(1-\mathscr{P}_{K}\right) L\right]^{-1}$ reduce $\mathscr{J}_{M K}$ and $\mathscr{I}_{K E}$ to the same operator. Hence, the functional (26) suggests that even crude approximations within the KEF will yield results superior to (or at least no worse than ) similar treatments within the MFK.

\section{THE INTRODUCTION OF SPECIFIC APPROXIMATIONS INTO THE PROJECTION OPERATOR FORMALISMS}

Approximations based upon perturbation expansions of the familiar time evolution operator $[s-i L]^{-1}$ are quite common in studies of many-body 
systems. It is difficult to introduce approximations into projection operator formalisms through such expansions, however, since very little is known about the behavior of the modified propagators $\left(s-i\left[1-\mathscr{P}_{M}\right) L\right]^{-1}$ and $\left[s-i\left(1-\mathscr{P}_{K}\right) L\right]^{-1}$.

On the other hand, it is not always necessary to resort to approximations for these modified propagators. The memory and collision kernels can be expressed formally in terms of correlation functions which evolve under the unmodified propagator $[s-i L]^{-1}$. The memory kernel $\widetilde{K}(s)$, for example, is related to the force autocorrelation function

$$
\tilde{k}(s) \equiv(\beta / 3 m)\left\langle\mathbf{F}_{1} \cdot[1 /(s-i L)] \mathbf{F}_{\mathbf{1}}\right\rangle
$$

through the identity

$$
\tilde{K}(s)=\tilde{k}(s)+(1 / s) \tilde{k}(s) \tilde{K}(s)
$$

Similarly, Akcasu(7) and Mazenko(8) have shown that the collision kernel satisfies an identity which, in the limit of long wavelengths, reduces to

$$
\tilde{\varphi}\left(\mathbf{p}, \mathbf{p}^{\prime}, s\right)=\tilde{\phi}\left(\mathbf{p}, \mathbf{p}^{\prime}, s\right)+(1 / s) \int d^{3} p^{\prime \prime} \tilde{\phi}\left(\mathbf{p}^{\prime \prime}, \mathbf{p}^{\prime}, s\right) \tilde{\varphi}\left(\mathbf{p}, \mathbf{p}^{\prime \prime}, s\right)
$$

where $\tilde{\phi}\left(\mathbf{p}, \mathbf{p}^{\prime}, s\right)$ evolves under the unmodified propagator,

$$
\tilde{\phi}\left(\mathbf{p}, \mathbf{p}^{\prime}, s\right) \equiv \frac{1}{M\left(\mathbf{p}^{\prime}\right)}\left\langle\mathbf{F}_{1} \cdot\left[\frac{\partial}{\partial \mathbf{p}_{1}} \delta\left(\mathbf{p}^{\prime}-\mathbf{p}_{1}\right)\right] \frac{1}{s-i L} \mathbf{F}_{\mathbf{1}} \cdot \frac{\partial}{\partial \mathbf{p}_{1}} \delta\left(\mathbf{p}-\mathbf{p}_{1}\right)\right\rangle
$$

Consequently, Eqs. (32) and (33) can be iterated to generate formal perturbation expansions for $\widetilde{K}(s)$ and $\tilde{\varphi}\left(\mathbf{p}, \mathbf{p}^{\prime}, s\right)$, respectively, in terms of the unmodified propagator. As Akcasu has pointed out, to lowest order in such expansions, $\widetilde{K}(s) \sim \tilde{k}(s)$ and $\tilde{\varphi}\left(\mathbf{p}, \mathbf{p}^{\prime}, s\right) \sim \tilde{\phi}\left(\mathbf{p}, \mathbf{p}^{\prime}, s\right)$. More precisely, if the Liouville operator is decomposed as

$$
L=L_{0}+\epsilon L_{1}
$$

where $\epsilon$ is a perturbation parameter, then a formal expansion of $[s-i L]^{-1}$ in powers of $\epsilon$ reveals that

$$
\tilde{K}(s)=\tilde{k}(s)+O\left(\epsilon^{3}\right)
$$

and

$$
\tilde{\varphi}\left(\mathbf{p}, \mathbf{p}^{\prime}, s\right)=\tilde{\phi}\left(\mathbf{p}, \mathbf{p}^{\prime}, s\right)+O\left(\epsilon^{3}\right)
$$

Equations (36) and (37) imply that the lowest-order terms of the generalized collision operators $\mathscr{J}_{M K}$ and $\mathscr{J}_{K E}$ are identical. This result, in conjunction with Eqs. (16) and (20), implies that the MKF and KEF necessarily 
predict different forms for the momentum autocorrelation function within the context of a lowest-order perturbation approximation. This difference has particularly serious consequences for approximations which utilize the Markovian $(s \rightarrow 0)$ behavior of the memory kernel.

Resibois et al. ${ }^{(1)}$ studied the memory kernel equation (2) in the context of a particular Markovian limit, the so-called weak-coupling limit. In this approximation the interparticle potential for the system is characterized by a parameter $\lambda$ which goes to zero in such a way that the product $\lambda^{2} t$ remains finite as $t \rightarrow \infty$. It is straightforward to demonstrate that, to lowest order in $\lambda$,

$$
K(t) \rightarrow K_{0}(t)=-\frac{\lambda^{2}}{3 m} \int \frac{d^{3} k}{(2 \pi)^{3}} k^{2} \tilde{V}(k) n \tilde{g}(k) \exp \left(-\frac{k^{2} t^{2}}{m \beta}\right)
$$

where $\tilde{V}(k)$ is the Fourier transform of the interparticle potential, $n$ is the average density of particles, and $\tilde{g}(k)$ is the static pair correlation function,

$$
n \tilde{g}(k) \equiv \sum_{\alpha=2}^{N}\left\langle\exp \left[i \mathbf{k} \cdot\left(\mathbf{x}_{1}-\mathbf{x}_{\alpha}\right)\right]\right\rangle
$$

The approximation (38) gives invalid results in the limit of long times, however, since the weak-coupling expansion for the memory kernel does not converge uniformly.

A weak-coupling expansion can be made for the collision kernel in the KEF as well. To lowest order in $\lambda$, the kinetic equation (17) becomes

$$
s \tilde{\boldsymbol{\Theta}}(\mathbf{p}, s)-\frac{1}{\beta} \frac{\partial}{\partial \mathbf{p}} \cdot \tilde{\Xi}(\mathbf{p}, s) \cdot\left(\frac{\partial}{\partial \mathbf{p}}+\frac{\beta}{m} \mathbf{p}\right) \tilde{\boldsymbol{\Theta}}(\mathbf{p}, s)=\mathbf{p} M(\mathbf{p})
$$

where

$$
\Xi(\mathbf{p}, t) \equiv \beta\left\langle\mathbf{F}_{1} \mathbf{F}_{12}\left\{\mathbf{x}_{1}-\mathbf{x}_{2}+(t / m)\left[\mathbf{p}-\mathbf{p}_{2}\right]\right\}\right\rangle
$$

and $\mathbf{F}_{12}$ is the force exerted upon the test particle by a single bath particle arbitrarily labeled " 2 ." The Markovian limit of Eq. (40) is just the linearized Fokker-Planck equation, which is the accepted weak-coupling description of Brownian motion.

It is apparent in this case that it is the presence of the additional projection operator $\mathscr{P}_{1}$ which leads to the incorrect Markovian results for the MKF. Specifically, $\mathscr{P}_{1}$ removes the p dependence from $\tilde{\Xi}(\mathbf{p}, s)$ in such a way that the field variable $\mathbf{p}$ is replaced by $\mathbf{p}_{1}$ before the ensemble average is taken. In that case

$$
\tilde{\Xi}(\mathbf{p}, s) \rightarrow \beta \tilde{K}(s) 1
$$

and the two formalisms become identical. 
The low-density approximation is another Markovian limit which is employed quite often. In this limit the particle density $n$ becomes infinitesimally small in such a way the product $n t$ remains finite as $t \rightarrow \infty$. Specifically, if the Liouville operator is decomposed as

$$
\begin{aligned}
i L= & \left\{\frac{1}{m} \mathbf{p}_{1} \cdot \frac{\partial}{\partial \mathbf{x}_{1}}+\frac{1}{m} \mathbf{p}_{2} \cdot \frac{\partial}{\partial \mathbf{x}_{2}}+\mathbf{F}_{12} \cdot\left(\frac{\partial}{\partial \mathbf{p}_{1}}-\frac{\partial}{\partial \mathbf{p}_{2}}\right)\right\} \\
& +\left\{\sum_{\alpha=3}^{N} \frac{1}{m} \mathbf{p}_{\alpha} \cdot \frac{\partial}{\partial \mathbf{x}_{\alpha}}\right\}+\left\{\frac{1}{2} \sum_{\alpha=3}^{N} \sum_{\substack{\gamma=1 \\
\neq \alpha}}^{N} \mathbf{F}_{\alpha \gamma} \cdot\left(\frac{\partial}{\partial \mathbf{p}_{\alpha}}-\frac{\partial}{\partial \mathbf{p}_{\gamma}}\right)\right\} \\
\equiv & \left\{i L_{12}\right\}+\left\{i L_{0, N-2}\right\}+\left\{i L_{R}\right\}
\end{aligned}
$$

then to lowest order in density,

$$
\begin{aligned}
\tilde{K}(s) \rightarrow \tilde{K}_{0}(s) & =\frac{\beta}{3 m}\left\langle\mathbf{F}_{1} \cdot \frac{1}{s-i L_{12}-i L_{0, N-2}} \mathbf{F}_{1}\right\rangle \\
& =\frac{\beta}{3 m} \int d^{3} p_{1} M\left(\mathbf{p}_{1}\right) \mathbf{p}_{1} \cdot\left\langle L \frac{1}{s-i L_{12}} L\right\rangle_{B} \mathbf{p}_{1}
\end{aligned}
$$

It has been shown ${ }^{(7-11)}$ that the operator $\left\langle L\left[1 /\left(s-i L_{12}\right)\right] L\right\rangle_{B}$ is related to the two-particle Liouville operator $L_{T P}$,

$$
i L_{T P} \equiv \frac{2}{m} \mathbf{q} \cdot \frac{\partial}{\partial \mathbf{r}}-\frac{\partial V}{\partial \mathbf{r}} \cdot \frac{\partial}{\partial \mathbf{q}}
$$

where

$$
\mathbf{r} \equiv \mathbf{x}_{1}-\mathbf{x}_{2}
$$

and

$$
\mathbf{q} \equiv \frac{1}{2}\left(\mathbf{p}_{\mathbf{1}}-\mathbf{p}_{2}\right)
$$

In terms of this two-particle operator, Eq. (44) becomes

$$
\tilde{K}_{0}(s)=\frac{4}{3} \frac{\beta}{m}\left(\frac{\beta}{\pi m}\right)^{3 / 2} n \int d^{3} r \int d^{3} q\left(\exp -\frac{\beta q^{2}}{m}\right) g(r) \frac{\partial V}{\partial \mathbf{r}} \cdot \frac{1}{s-i L_{T P}} \frac{\partial V}{\partial \mathbf{r}}
$$

It can be shown in similar fashion that to lowest order in density the longwavelength limit of the collision kernel is

$$
\begin{aligned}
\tilde{\varphi}\left(\mathbf{p}, \mathbf{p}^{\prime}, s\right) \rightarrow \tilde{\varphi}_{0}\left(\mathbf{p}, \mathbf{p}^{\prime}, s\right) \\
=\frac{n}{M\left(\mathbf{p}^{\prime}\right)}\left(\frac{\beta}{\pi m}\right)^{3} \frac{\partial}{\partial \mathbf{p}} \frac{\partial}{\partial \mathbf{p}^{\prime}} \cdot \int d^{3} r \int d^{3} q \int d^{3} \alpha\left(\exp -\frac{\beta\left(\alpha^{2}+q^{2}\right)}{m}\right) \\
\quad \times\left\{g(r) \frac{\partial V}{\partial \mathbf{r}} \delta\left(\mathbf{p}^{\prime}-\boldsymbol{\alpha}-\mathbf{q}\right) \frac{1}{s-i L_{T P}} \frac{\partial V}{\partial \mathbf{r}} \delta(\mathbf{p}-\boldsymbol{\alpha}+\mathbf{q})\right\}
\end{aligned}
$$


where

$$
\alpha \equiv \frac{1}{2}\left(\mathbf{p}_{1}+\mathbf{p}_{2}\right)
$$

In the Markovian limit the operator $\left\langle L\left[1 /\left(s-i L_{\mathrm{1} 2}\right)\right] L\right\rangle_{B}$ reduces to the linearized Boltzmann operator $J_{B}{ }^{(9-12)}$ In that case, the KEF predicts that

$$
\widetilde{\Phi}(s)=(\beta / 3 m) \int d^{3} p_{1} M\left(\mathbf{p}_{1}\right) \mathbf{p}_{1} \cdot\left[1 /\left(s+J_{B}\right)\right] \mathbf{p}_{1}
$$

while the MKF predicts that

$$
\tilde{\Phi}(s)=1 /\left[s+\widetilde{K}_{0}(0)\right]
$$

where

$$
\widetilde{K}_{0}(s)=(\beta / 3 m) \int d^{3} p_{1} M\left(\mathbf{p}_{1}\right) \mathbf{p}_{1} \cdot J_{B} \mathbf{p}_{1}
$$

Thus the KEF again yields results which are quite different from-and, in fact, superior to-those given by the MKF in the context of a low-order Markovian approximation.

Another perturbation expansion which is closely related to the original conception of Brownian motion is the short-memory approximation. (13) In this scheme the Liouville operator is decomposed into two parts,

$$
\begin{aligned}
i L & =\left\{\sum_{\alpha=1}^{N} \frac{1}{m} \mathbf{p}_{\alpha} \cdot \frac{\partial}{\partial \mathbf{x}_{\alpha}}+\sum_{\gamma=2}^{N} \mathbf{F}_{\gamma} \cdot \frac{\partial}{\partial \mathbf{p}_{\gamma}}\right\}+\left\{\mathbf{F}_{\mathbf{1}} \cdot \frac{\partial}{\partial \mathbf{p}_{1}}\right\} \\
& \equiv\left\{i L_{1 B}\right\}+\left\{i L_{F}\right\}
\end{aligned}
$$

The operator $i L_{1 B}$ contains the details of the free streaming of all $N$ particles and of all coupling effects upon bath particles. The operator $i L_{F}$, on the other hand, describes only the effect of the bath particles upon the test particle.

It is straightforward to demonstrate that

$$
\mathscr{P}_{M} L_{1 B}=0
$$

and hence the modified propagator may be expanded as

$$
\frac{1}{s-i\left(1-\mathscr{P}_{M}\right) L}=\frac{1}{s-i L_{1 B}} \sum_{j=0}^{\infty}\left\{i\left(1-\mathscr{P}_{M}\right) L_{F} \frac{1}{s-i L_{1 B}}\right\}^{j}
$$

If only the first term in this expansion is retained, the memory kernel is approximated as

$$
K(t) \rightarrow K_{0}(t)=-\frac{1}{3 m} \int \frac{d^{3} k}{(2 \pi)^{3}} k^{2} \tilde{V}(k) \tilde{g}_{d}(k, t) \exp \left(\frac{-k^{2} t^{2}}{m \beta}\right)
$$


The function $\tilde{g}_{d}(k, t)$,

$$
\left.\left.\tilde{g}_{d}(k, t) \equiv \sum_{\alpha=2}^{N}\left\langle\exp \left(i \mathbf{k} \cdot \mathbf{x}_{1}\right) \exp \left(i t L_{1 B}\right) \exp \right)-i \mathbf{k} \cdot \mathbf{x}_{\alpha}\right)\right\rangle
$$

is closely related to Van Hove's distinct density correlation function, (14)

$$
\widetilde{G}_{\tilde{d}}(k, t) \equiv \sum_{\alpha=2}^{N}\left\langle\exp \left(i \mathbf{k} \cdot \mathbf{x}_{1}\right) \exp (i t L) \exp \left(-i \mathbf{k} \cdot \mathbf{x}_{\alpha}\right)\right\rangle
$$

If $\tilde{g}_{d}$ is approximated as $\tilde{G}_{d}$, the lowest-order short-memory approximation correctly describes the short-time behavior of the momentum autocorrelation function

$$
\Phi_{0}(t) \underset{t \rightarrow 0}{\longrightarrow} 1-(1 / 3 m)\left\langle\nabla^{2} V\right\rangle\left(t^{2} / 2 !\right)+O\left(t^{4}\right)
$$

However, this same approximation predicts a long-time decay proportional to $t^{-5}$ rather than the $t^{-3 / 2}$ behavior revealed by simulations of molecular dynamics. ${ }^{(15-17)}$

The root of this discrepancy lies within the approximation itself. The retention of $i L_{1 B}$ allows the motions of the bath particles to be influenced by the movement of the test particle, but the elimination of $i L_{F}$ means that the test particle is not, in turn, affected by these motions of the bath particles.

The short-memory approximation can be introduced into the KEF as well. If the modified propagator $\left[s-i\left(1-\mathscr{P}_{K}\right) L\right]^{-1}$ is expanded in analogy with Eq. (56), the lowest-order approximation to the collision kernel is

$$
\begin{aligned}
\tilde{\varphi}\left(\mathbf{p}, \mathbf{p}^{\prime}, s\right) \rightarrow & \tilde{\varphi}_{0}\left(\mathbf{p}, \mathbf{p}^{\prime}, s\right) \\
& =\frac{1}{M\left(\mathbf{p}^{\prime}\right)} \frac{\partial}{\partial \mathbf{p}^{\prime}} \cdot M\left(\mathbf{p}^{\prime}\right) \cong(\mathbf{p}, s) \cdot \frac{\partial}{\partial \mathbf{p}} \delta\left(\mathbf{p}-\mathbf{p}^{\prime}\right)
\end{aligned}
$$

where now

$$
\tilde{\Xi}(\mathbf{p}, s)=\int\left[d^{3} k /(2 \pi)^{3}\right] \mathbf{k} \mathbf{k}|\tilde{V}(k)|^{2} \tilde{g}_{d}(k, s+[i / m] \mathbf{k} \cdot \mathbf{p})
$$

To lowest order, $\tilde{g}_{d}(k, s)$ can be approximated by its self-consistent field representation,

$$
\tilde{g}_{d}(k, s) \rightarrow \frac{n}{D^{+}(k, s)} \int d^{3} p^{\prime \prime} M\left(\mathbf{p}^{\prime \prime}\right) \frac{1}{s+(i / m) \overline{\mathbf{k}} \cdot \mathbf{p}^{\prime \prime}}
$$

where the function $D^{+}(k, s)$ is given by

$$
D^{+}(k, s)=1+i \frac{\beta}{m} \int d^{3} p^{\prime \prime} M\left(\mathbf{p}^{\prime \prime}\right) \frac{1}{s+(i / m) \mathbf{k} \cdot \mathbf{p}^{\prime \prime}}:\left.\tilde{V}(k)\right|^{2} \mathbf{k} \cdot \mathbf{p}^{\prime \prime}
$$


If a tensor $\mathbf{Q}\left(\mathbf{p}, \mathbf{p}^{\prime}\right)$ is defined as

$\mathrm{Q}\left(\mathbf{p}, \mathbf{p}^{\prime}\right) \equiv n \int \frac{d^{3} k}{(2 \pi)^{3}} \mathbf{k k}|\tilde{V}(k)|^{2} \frac{1}{\left|D^{+}(k, i \mathbf{k} \cdot \mathbf{p} / m)\right|^{2}} \delta\left(\frac{1}{m} \mathbf{k} \cdot\left[\mathbf{p}-\mathbf{p}^{\prime}\right]\right)$

then in the Markovian limit

$$
\tilde{\Xi}(\mathbf{p}, s) \underset{s \rightarrow 0}{\longrightarrow} \int d^{3} p^{\prime} M\left(\mathbf{p}^{\prime}\right) \mathrm{Q}\left(\mathbf{p}, \mathbf{p}^{\prime}\right)
$$

Consequently, in this limit the kinetic equation (17) reduces to the familiar linearized Balescu-Lennard equation,

$$
s \tilde{\mathbf{\Theta}}(\mathbf{p}, s)+\frac{\partial}{\partial \mathbf{p}} \cdot \int d^{3} p^{\prime} M\left(\mathbf{p}^{\prime}\right) \mathbf{Q}\left(\mathbf{p}, \mathbf{p}^{\prime}\right) \cdot\left(\frac{\partial}{\partial \mathbf{p}}+\frac{\beta}{m} \mathbf{p}\right) \tilde{\mathbf{\Theta}}(\mathbf{p}, s)=\mathbf{p} M(\mathbf{p})
$$

This equation can be regarded, in a sense, as a generalization of the linearized Fokker-Planck equation which includes the effect of wave-particle interactions.

Another method for calculating first-order approximations to the memory and collision kernels is to replace the true modified propagator by a propagator which describes the interactions among particles in a general or "averaged" sense. Such an approximation is conceptually related to such quantum mechanical methods as the random-phase approximation. In this approach an attempt is made to solve explicitly for a function whose time evolution is governed by the approximate propagator. The general form of the equations which describe such a function is

$$
\left(s-i L_{A}\right) \tilde{\Psi}_{M}\left(\Gamma_{N}, s\right)=M\left(\mathbf{p}_{1}\right) \mathbf{F}_{1}
$$

in the MFK and

$$
\left(s-i L_{A}\right) \tilde{\psi}_{K}\left(\Gamma_{N}, \mathbf{p}, s\right)=M\left(\mathbf{p}_{1}\right) \mathbf{F}_{1} \cdot\left(\partial / \partial \mathbf{p}_{1}\right) \delta\left(\mathbf{p}-\mathbf{p}_{1}\right)
$$

in the KEF. Once $\tilde{\psi}_{M}$ and $\tilde{\psi}_{K}$ are known the approximate memory and collision kernels can be calculated from the relations

$$
\begin{aligned}
\tilde{K}_{0}(s) & =\frac{\beta}{3 m}\left\langle\mathbf{F}_{1} \cdot \frac{1}{M\left(\mathbf{p}_{1}\right)} \psi_{M}\left(\Gamma_{N}, s\right)\right\rangle \\
& =-\frac{1}{3 m}\left\langle\frac{1}{M\left(\mathbf{p}_{1}\right)} \frac{\partial}{\partial \mathbf{x}_{1}} \cdot \psi_{M}\left(\Gamma_{N}, s\right)\right\rangle
\end{aligned}
$$

and

$$
\begin{aligned}
\tilde{\varphi}_{\mathbf{0}}\left(\mathbf{p}, \mathbf{p}^{\prime}, s\right) & =\frac{1}{M\left(\mathbf{p}^{\prime}\right)}\left\langle\mathbf{F}_{1} \cdot\left[\frac{\partial}{\partial \mathbf{p}_{1}} \delta\left(\mathbf{p}^{\prime}-\mathbf{p}_{1}\right)\right] \frac{1}{M\left(\mathbf{p}_{1}\right)} \psi_{K}\left(\Gamma_{N}, \mathbf{p}, s\right)\right\rangle \\
& =-\frac{1}{\beta M\left(\mathbf{p}^{\prime}\right)}\left\langle\frac{1}{M\left(\mathbf{p}_{1}\right)}\left[\frac{\partial}{\partial \mathbf{p}_{1}} \delta\left(\mathbf{p}^{\prime}-\mathbf{p}_{1}\right)\right] \cdot \frac{\partial}{\partial \mathbf{x}_{1}} \psi_{K}\left(\Gamma_{N}, \mathbf{p}, s\right)\right\rangle
\end{aligned}
$$


since, as shown in Section 2, the modified propagators commute with the Maxwellian.

One simple well-known operator which lends itself to such an approximation is the Bhatnagar-Gross-Krook model ${ }^{(18)}$ of the Lorentz-Boltzmann operator,

$$
\begin{aligned}
i L_{L B} \tilde{\mathbf{G}}\left(\Gamma_{N}, s\right) \equiv & -(1 / m) \mathbf{p}_{1} \cdot\left(\partial / \partial \mathbf{x}_{1}\right) \tilde{\mathbf{G}}\left(\Gamma_{N}, s\right)-\nu_{\theta} \tilde{\mathbf{G}}\left(\Gamma_{N}, s\right) \\
& +\nu_{c} M\left(\mathbf{p}_{1}\right) \int d^{3} \hat{p}_{\mathbf{1}} \tilde{\mathbf{G}}\left(\hat{\Gamma}_{N}, s\right)
\end{aligned}
$$

where $\nu_{c}$ is the mean collision frequency. In the MKF the Fourier transform of the function which evolves under this propagator is

$\tilde{\Psi}_{M}\left(\mathbf{k}, \mathbf{p}_{1}, s\right)=\frac{M\left(\mathbf{p}_{1}\right)}{\Lambda(k, s)} \frac{1}{s-(i / m) \mathbf{k} \cdot \mathbf{p}_{1}+v_{c}} i \mathbf{k} \tilde{V}(k) \sum_{\alpha=2}^{N} \exp -i \mathbf{k} \cdot \mathbf{x}_{\alpha}$

where

$$
A(k, s) \equiv 1-\nu_{c} \int d^{3} p^{\prime \prime} M\left(\mathbf{p}^{\prime \prime}\right) \frac{1}{s-(i / m) \mathbf{k} \cdot \mathbf{p}^{\prime \prime}+\nu_{c}}
$$

Equations (70) and (73) imply that

$$
\tilde{K}_{0}(s)=-\frac{n}{3 m} \int \frac{d^{3} k}{(2 \pi)^{3}} k^{2} \tilde{V}(k) \tilde{g}(k) \frac{1-\Lambda(k, s)}{\nu_{c} \Lambda(k, s)}
$$

This model, like many others, correctly predicts the short-time behavior of the momentum autocorrelation function. For very short times and long wavelengths it is obvious that

$$
A(k, s) \underset{k \rightarrow 0, s \rightarrow \infty}{\longrightarrow} 1-(1 / s) \nu_{c}
$$

and hence

$$
\widetilde{K}_{0}(s) \underset{s \rightarrow \infty}{\longrightarrow}-\frac{n}{3 m} \int \frac{d^{3} k}{(2 \pi)^{3}} k^{2} \tilde{V}(k) \tilde{g}(k) \frac{1}{s} \equiv \delta^{2} / s
$$

where $\delta^{2}>0$, since $\tilde{g}(k)$ approaches a negative constant for small $k$. Consequently, Eqs. (15) and (77) imply that, to lowest order,

$$
\Phi_{0}(t) \underset{t \rightarrow 0}{\rightarrow} \cos (\delta t)
$$

At long times, on the other hand, the dominant contribution to the integral in Eq. (72) comes from the region near the origin in $\mathbf{k}$ space. But it is known that

$$
v_{c} \Lambda(k, s) \underset{k \rightarrow 0, s \rightarrow 0}{\longrightarrow} s+k^{2} D_{s}
$$


where $D_{s}$ is the coefficient of self-diffusion for this model,

$$
D_{s} \equiv\left(1 / 3 v_{c}\right)\left\langle P_{1}^{2} / m^{2}\right\rangle=1 / m \beta \nu_{c}
$$

Consequently, Eq. (75) implies that for long times

$$
K_{0}(t) \underset{t \rightarrow \infty}{\longrightarrow}-\frac{n}{3 m} \int \frac{d^{3} k}{(2 \pi)^{3}} k^{2} \tilde{V}(k) \tilde{g}(k) \exp \left(-k^{2} D_{s} t\right)
$$

This result will be discussed in somewhat greater detail below.

The introduction of this approximation into the KEF yields a kinetic equation which is so complicated that further approximations would have to be introduced before it could be solved analytically. The introduction of these further approximations would prevent a direct comparison of the results from the two formalisms, however.

The collective motions of the bath can be emphasized even more heavily if the modified propagator is approximated as the linearized Boltzmann operator. Although the resulting equation can be solved only for certain modeled approximations to this operator (e.g., the BGK model), the general structure of these solutions is such that

$$
\int d^{3} p_{1} \tilde{\psi}_{M}\left(\mathbf{k}, \mathbf{p}_{1}, s\right)=i \mathbf{k} \tilde{V}(k) \frac{\chi_{B}(\mathbf{k}, s)}{\Lambda_{B}(k, s)} \sum_{\alpha=1}^{N} \exp \left(-i \mathbf{k} \cdot \mathbf{x}_{\alpha}\right)
$$

where $\Lambda_{B}(k, s)$ is the dispersion law which characterizes the Boltzmann operator and $\chi_{B}(\mathbf{k}, s)$ is a function whose exact form depends upon the particular model adopted.

It is well known that $\Lambda_{B}(k, s)$ assumes a hydrodynamic form in the limit of small $k$ and $s,{ }^{(19-21)}$

$\Lambda_{B}(k, s) \underset{k \rightarrow 0, s \rightarrow 0}{\longrightarrow}$ const $\times\left(s+i c k+\Gamma k^{2}\right)\left(s-i c k+\Gamma k^{2}\right)\left(s+D_{T} k^{2}\right)$

where $c$ is the isothermal speed of sound, $\Gamma$ is the coefficient of sound attenuation, and $D_{r}$ is the thermal diffusivity. For liquids, for example, viscous effects predominate in such a way that

$$
\Gamma \rightarrow \nu \ll D_{T}
$$

where $v$ is the kinematic viscosity. Furthermore, the oscillatory contributions from the sound propagation average to zero for small $k$ and $s$. The memory kernel therefore assumes the asymptotic form

$$
K_{0}(t) \underset{t \rightarrow \infty}{\longrightarrow}-\frac{n}{3 m} \int \frac{d^{3} k}{(2 \pi)^{3}} k^{2} \tilde{V}(k) \tilde{g}(k) \exp \left(-k^{2} \nu t\right)
$$


This result is identical to Eq. (81) except that the self-diffusion coefficient $D_{s}$ has been replaced by the kinematic viscosity $\nu$. Likewise, the kinetic equation corresponding to a particular model for the linearized Boltzmann operator will be very similar to the results for the Lorentz Boltzmann operator but with $\Lambda(k, s)$ replaced by $\Lambda_{B}(k, s)$.

It is not surprising that it is $\nu$ rather than $D_{s}$ which appears in Eq. (85). The linearized Boltzmann operator emphasizes collective motions, while the Lorentz-Boltzmann operator stresses self-diffusion. Equations (81) and (85) suggest that the memory kernel may actually evolve as

$$
K_{0}(t) \underset{t \rightarrow \infty}{\rightarrow}-\frac{n}{3 m} \int \frac{d^{3} k}{(2 \pi)^{3}} k^{2} \tilde{V}(k) \tilde{g}(k) \exp \left[-k^{2}\left(\nu+D_{s}\right) t\right]
$$

A similar conclusion about the momentum autocorrelation function itself has been reached by a number of researchers ${ }^{(9,16,17,22-25)}$

$$
\Phi(t) \underset{t \rightarrow \infty}{\longrightarrow} \text { const } \times\left[\left(v+D_{s}\right) t\right]^{-3 / 2}
$$

Equation (86) offers an exceedingly simple solution for the memory kernel for any system characterized by a Fourier-transformable interparticle potential. More surprisingly, it suggests that the momentum autocorrelation function may decay according to different power laws for systems characterized by different interparticle potentials. ${ }^{(26)}$

\section{SUMMARY}

In this paper the compatibility of two projection operator formalisms with certain approximation schemes has been compared within the context of Brownian motion. The memory kernel formalism (MKF) is based upon a memory kernel equation for the momentum autocorrelation function itself, while the kinetic equation formalism (KEF) is based upon a kinetic equation for its phase-space generalization.

The difference in the two formalisms was shown to be due to the presence of an additional projection operator in the MKF. This operator, denoted as $\mathscr{P}_{1}$, effectively reduces the phase-space description inherent in the KEF to a configuration-space description and causes the MKF to yield incorrect results in the long-time limit of certain low-order perturbation approximations. Furthermore, a variational formulation of the KEF demonstrated that for even fairly crude approximations the KEF yields results superior to (or at least no worse than) similar treatments within the MKF.

The results from standard weak-coupling and low-density approximations were reviewed for both formalisms. The results from the short- 
memory approximation in the MKF were reviewed as well, and it was demonstrated that the Markovian limit of this approximation reduced to the KEF description to the linearized Balescu-Lennard equation. The Markovian limit of each of these approximations gave meaningful results in the KEF, but the corresponding results in the MKF were in contradiction to the asymptotic $t^{-3 / 2}$ decay of the momentum autocorrelation function recently revealed by computer simulations of molecular dynamics.

The MKF did yield very interesting results for approximations based on operators which describe particle interactions in an "averaged" sense. In particular, results from approximations based on the Lorentz-Boltzmann and linearized Boltzmann operators suggest that the asymptotic behavior of the kernel is given by

$$
K(t) \underset{t \rightarrow \infty}{\longrightarrow}-\frac{n}{3 m} \int \frac{d^{3} k}{(2 \pi)^{3}} k^{2} \tilde{V}(k) \tilde{g}(k) \exp \left[-k^{2}\left(\nu+D_{s}\right) t\right]
$$

This result, in turn, suggests that contrary to previous expectations, the momentum autocorrelation function decays according to different power laws for systems characterized by different interparticle potentials.

\section{APPENDIX}

The collision kernel is defined as

$$
\varphi\left(\mathbf{p}, \mathbf{p}^{\prime}, t\right) \equiv \frac{1}{M\left(\mathbf{p}^{\prime}\right)}\left\langle\mathbf{F}_{1} \cdot\left[\frac{\partial}{\partial \mathbf{p}_{1}} \delta\left(\mathbf{p}^{\prime}-\mathbf{p}_{1}\right)\right] e^{i t\left(1-\mathscr{P}_{K}\right) L} \mathbf{F}_{1} \cdot \frac{\partial}{\partial \mathbf{p}_{1}} \delta\left(\mathbf{p}-\mathbf{p}_{1}\right)\right\rangle
$$

However, the operator $\left(1-\mathscr{P}_{K}\right)$ is Hermitian on the space of functions orthogonal to $\mathbf{p}_{\mathbf{1}}$, and so Eq. (A.1) may be rewritten as

$$
\begin{aligned}
& \varphi\left(\mathbf{p}, \mathbf{p}^{\prime}, t\right)=\frac{1}{M\left(\mathbf{p}^{\prime}\right)}\left\langle\mathbf{F}_{1} \cdot\left[\frac{\partial}{\partial \mathbf{p}_{1}} \delta\left(\mathbf{p}-\mathbf{p}_{1}\right)\right] e^{-i t\left(1-\mathscr{O}_{K}\right) L} \mathbf{F}_{1} \cdot \frac{\partial}{\partial \mathbf{p}_{1}} \delta\left(\mathbf{p}^{\prime}-\mathbf{p}_{1}\right)\right\rangle \\
& =\left[\frac{M(\mathbf{p})}{M\left(\mathbf{p}^{\prime}\right)}\right] \frac{1}{M(\mathbf{p})}\left\langle\mathbf{F}_{1} \cdot\left[\frac{\partial}{\partial \mathbf{p}_{1}} \delta\left(\mathbf{p}-\mathbf{p}_{1}\right)\right] e^{i t\left(1-\mathscr{P}_{K}\right) L} \mathbf{F}_{1} \cdot \frac{\partial}{\partial \mathbf{p}_{1}} \delta\left(\mathbf{p}^{\prime}-\mathbf{p}_{1}\right)\right\rangle^{*} \\
& =\frac{M(\mathbf{p})}{M\left(\mathbf{p}^{\prime}\right)} \varphi^{*}\left(\mathbf{p}^{\prime}, \mathbf{p}, t\right)
\end{aligned}
$$

But $\varphi$ is obviously real, and hence it satisfies the detailed balance relation

$$
M\left(\mathbf{p}^{\prime}\right) \varphi\left(\mathbf{p}, \mathbf{p}^{\prime}, t\right)=M(\mathbf{p}) \varphi\left(\mathbf{p}^{\prime}, \mathbf{p}, t\right)
$$




\section{ACKNOWLEDGMENTS}

The authors gratefully acknowledge many fruitful discussions with A. Z. Akcasu and C. M. Bost, Ir. In addition, one of the authors (R.D.M.) wishes to acknowledge financial support from an AEC-NSE fellowship during a period in which much of this research was being carried out.

\section{REFERENCES}

1. P. Resibois, J. Brocas, and G. Decan, J. Math. Phys. 10:964 (1969).

2. R. Zwanzig, in Lectures in Theoretical Physics, Vol. III, W. E. Brittin, W. B. Downs, and J. Downs, eds., Interscience, New York (1961).

3. R. Zwanzig, J. Chem. Phys. 33:1338 (1960).

4. R. Zwanzig, Phys. Rev. 124:983 (1961).

5. H. Mori, Progr. Theoret. Phys. (Kyoto) 33:423 (1965).

6. N. C. Francis, J. C. Stewart, L. S. Bohl, and T. J. Krieger, in Second International Conference on the Peaceful Uses of Atomic Energy, P/627, Geneva, 1958.

7. A. Z. Akcasu, Phys. Rev. A 7:182 (1973).

8. G. F. Mazenko, Phys. Rev. A 7:222 (1973).

9. J. R. Dorfman and E. G. D. Cohen, Phys. Rev. Letters 25:1257 (1970).

10. G. F. Mazenko, Phys. Rev. A 3:2121 (1971).

11. G. F. Mazenko, Phys. Rev. A 5:2545 (1972).

12. M. H. Ernst, Lectures in Theoretical Physics, Vol. 9C, Gordon and Breach, New York (1967).

13. N. Corngold and J. J. Duderstadt, Phys. Rev. A 2:836 (1970).

14. L. Van Hove, Phys. Rev. 95:249 (1954).

15. B. J. Alder and T. E. Wainwright, Phys. Rev. Letters 18:988 (1967).

16. B. J. Alder and T. E. Wainwright, Phys. Rev. A 1:18 (1970),

17. B. J. Alder, D. M. Gass, and T. E. Wainwright, J. Chem. Phys. 53:3813 (1970).

18. P. L. Bhatnagar, E. P. Gross, and M. Krook, Phys. Rev. 94:511 (1954).

19. S. Chapman and T. G. Cowling, Mathematical Theory of Non-Uniform Gases, 3rd ed., Cambridge University Press, Cambridge (1960).

20. R. Mountain, Rev. Mod. Phys. 38:205 (1966).

21. M. Bixon, J. R. Dorfman, and K. C. Mo, Phys. Fluids 14:1049 (1971) and references contained therein.

22. M. H. Ernst, E. H. Hauge, and J. M. J. van Leeuwen, Phys. Rev. Letters 25:1254 (1970).

23. M. H. Ernst, E. H. Hauge, and J. M. J. van Leeuwen, Phys. Rev. A 4:2055 (1971).

24. B. J. Alder and T. E. Wainwright, J. Phys. Soc. Japan (Suppl). 26:267 (1968).

25. G. F. Mazenko, Phys. Rev. A 7:209 (1973).

26. R. D. Mosteller and J. J. Duderstadt, to be published. 